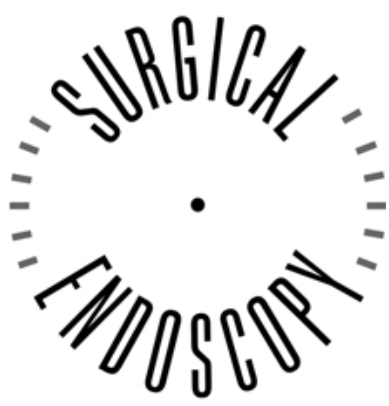

and Other Interventional Techniques

\title{
Endosonography of upper gastrointestinal tract cancer on demand using miniprobes or endoscopic ultrasound
}

\author{
M. Hünerbein, C. Ulmer, T. Handke, P. M. Schlag
}

[Surg Endosc (2003) 17: 615-619, DOI: 10.1007/s00464-002-8622-3]

The authors would like to change the captions for Tables 1 and 2 on page 617. The correct captions are:

Table 1. Correlation between preoperative assessment of tumor infiltration by endoscopic ultrasound (uT) and histopathology (pT) in 97 patients with esophagogastric cancer

Table 2. Correlation between preoperative assessment of tumor infiltration by miniprobe ultrasound (uT) and histopathology (pT) in 76 patients with esophagogastric cancer 